\title{
Estudio de la satisfacción de los estudiantes universitarios con asignaturas de contenido cuantitativo
}

\section{Study of the undergraduated student satisfaction at quantitative content subjects}

\author{
Ana M. Martin Caraballo. \\ Universidad Pablo de Olavide (UPO). \\ ammarcar@upo.es \\ Concepción Paralera Morales. \\ Universidad Pablo de Olavide (UPO). \\ cparmor@upo.es \\ José M. Ramírez Hurtado. \\ Universidad Pablo de Olavide (UPO). \\ imramhur@upo.es
}

\section{RESUMEN.}

La implantación del Espacio Europeo de Educación Superior obligó, hace ya unos años, a realizar numerosos cambios en la enseñanza universitaria. En este nuevo sistema, ya totalmente implantado en la actualidad, hace que sea el alumno el verdadero protagonista de su aprendizaje y que el profesor solo sea un mero ayudante de ese proceso. Así, el alumno tiene una participación activa y continua en el sistema. Por este motivo, creemos que el estudio de la satisfacción de los alumnos debe convertirse en un objetivo primordial, ya que nos llevará a detectar posibles anomalías en el desarrollo de la docencia. por ello, uno de los principales objetivos de este trabajo es tratar de medir la satisfacción de los estudiantes universitarios con asignaturas de contenido cuantitativo impartidas en grados de facultades de empresa. Además, se pretende estudiar si hay diferencias en la percepción que tienen los estudiantes sobre la docencia de estas asignaturas en distintas universidades españolas, utilizando para ello una variante del modelo tradicional de importancia-valoración. Los resultados muestran que, de forma general, los estudiantes presentan un mínimo grado de insatisfacción con la totalidad de los atributos, si bien, el grado de satisfacción tampoco es elevado. Por otro lado, el estudio mostrará que entre las distintas universidades existen diferencias significativas en la percepción que tienen los estudiantes de algunos aspectos de la docencia de estas asignaturas.

\section{PALABRAS CLAVE.}

Satisfacción; universidad; docencia; análisis de importancia-valoración.

\section{ABSTRACT.}

After the implementation of the European Higher Education Area many changes has been carried out at University. With this new system the students become a main character of themselves learning due to their active and continued participation in the system. Thus, the study of student satisfaction is a main objective in order to detect some defects in the

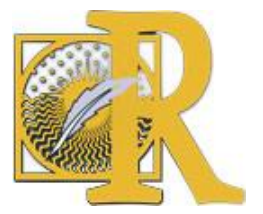


development of the teaching. Also, one of our goals is to study if there are differences in the perception that the students have about the teaching of these subjects in different Spanish universities. In order to do this, a variant of the traditional importance-valuation model will be used. This paper deals with the measure of the satisfaction of the undergraduate students having quantitative content subjects at Business Faculties. In order to do this, a variation of the traditional importance-performance analysis is used. In general, the results show that students have a minimum degree of dissatisfaction with all the attributes, although the degree of satisfaction is not too high. Furthermore, between the different universities there exist significant differences when we study the perception (and understanding) of the students of some aspects of the teaching of these subjects.

\section{KEY WORDS.}

Satisfaction, Higher Education, Teaching, Importance-Valuation Model.

\section{Introducción.}

El Espacio Europeo de Educación Superior es un ambicioso y complejo plan que han puesto en marcha los países del viejo continente para favorecer en materia de educación la convergencia europea. Se trata, sin lugar a dudas, de uno de los principales retos que los países de la Unión Europea plantearon a partir del año 1998 con la Declaración de la Sorbona de 1998 y la Declaración de Bolonia de 1999 suscrita por los ministros europeos de educación.

El proceso de convergencia hacia un Espacio Europeo de Educación Superior dirige el foco de sus actuaciones a construir un sistema de grados académicos reconocibles y comparables a nivel europeo, con objeto a fomentar la movilidad de los estudiantes, docentes e investigadores, garantizando en todo momento la calidad de la enseñanza a nivel superior.

Todo este proceso ha supuesto no sólo un profundo cambio de tipo estructural, centrado en adecuar determinados rasgos formales comunes a todas las instituciones de educación superior (Jacobs y Van der Ploeg, 2006), sino igualmente en la adopción de un enfoque distinto de la docencia (Tomusk, 2006; Escorcia et al., 2007; Herrera et al., 2008).

El informe "Student Centered Learning: Time for a New Paradigm in Education" (Esu, 2010) muestra con claridad cómo esta reforma no exige solamente una actualización de los planes de estudio, sino que plantea la necesidad de que las instituciones adapten su modelo educativo para impulsar este cambio hacia este nuevo paradigma. Por todo ello, las universidades se han visto obligadas a diseñar e implementar un nuevo modelo docente para garantizar los principios de esta reforma (Froment et al., 2010). Este cambio de cultura docente en la educación superior en Europa, propiciado por el desarrollo de las directrices del Plan de Bolonia, ha supuesto la puesta en marcha de numerosas propuestas de innovación docente con el fin de mejorar la actividad realizada por los profesores (Mauri et al., 2008). Como consecuencia de ello, ha surgido de forma paralela y en la misma intensidad, una necesidad de evaluar la calidad de las mismas.

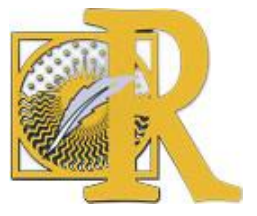

Fecha de recepción: 15-01-2020 Fecha de aceptación: 16-02-2020 
Con estas reformas la dedicación del alumnado a las diferentes materias cobra un gran protagonismo por dos razones fundamentales (Learreta et al., 2006). Por un lado, la carga de trabajo que asume el estudiante pasa a ser un eje fundamental en el nuevo sistema. Esta carga puede ser de diferente naturaleza: lecciones magistrales, trabajos prácticos, prácticas de informática, trabajo personal, exámenes u otras evaluaciones. Por tanto, la carga de trabajo del estudiante se convierte en la nueva unidad de medida que toma el crédito. Por otro lado, se plantea un sistema de enseñanza-aprendizaje en el que el alumno se convierte en el verdadero protagonista, por su participación activa y continua en el mismo.

La estructura de muchos sistemas educativos se caracteriza por ser heterogénea. Hay una gran diversidad de titulaciones y materias, que presentan contenidos diferentes y que hacen que la docencia deba concretarse con modelos y propuestas específicas. Todo ello hace aconsejable que la investigación se centre en el estudio de materias afines para poder disminuir el sesgo que la heterogeneidad puede provocar en los resultados. Este trabajo trata de seguir esta línea, analizando únicamente los datos de materias relacionadas con asignaturas cuantitativas de universidad.

Este trabajo contribuye a la literatura existente sobre el estudio de la satisfacción de los estudiantes universitarios con la docencia que reciben. De forma concreta, el objetivo de este trabajo es medir la satisfacción que muestra el alumnado con asignaturas del ámbito cuantitativo. Con él se pretenden identificar cuáles son los atributos de la docencia que provocan una mayor satisfacción en los alumnos y cuáles son los que provocan una mayor insatisfacción. De esta forma se pretende identificar aquellos factores que presentan una deficiencia en el desarrollo de la docencia y que, por tanto, deben ser corregidos para la mejora de la calidad de la enseñanza.

Para conseguir este objetivo, en este trabajo se va a utilizar una variante del análisis de importancia-valoración de Martilla y James (1977), propuesta por algunos autores (Ábalo et al., 2006; Picón et al., 2011). Hay que tener en cuenta que el paradigma de importanciavaloración es el camino más apropiado para medir la calidad de servicio en educación (Joseph and Joseph, 1997). Los resultados derivados de esta técnica proporcionan una estimable información para el desarrollo de estrategias educacionales.

La justificación de la realización de este estudio se encuentra en varias razones. En primer lugar, los estudiantes son algo más que simples "productos" de la enseñanza superior (Nale et al., 2000). Ellos tratan de satisfacer sus necesidades educacionales, por lo que es importante identificar sus percepciones sobre la docencia recibida y medir su nivel de satisfacción. Por otro lado, esta información constituye un gran valor para la institución universitaria ya que es de gran importancia para poder realizar una planificación docente más eficaz.

El trabajo se estructurará de la siguiente manera. Después de la introducción, en el segundo epígrafe se detallan los antecedentes, evolución y momento actual del EEES. En el tercer epígrafe se hace una revisión de la literatura en el ámbito de la satisfacción del estudiante universitario. A continuación, se detalla la metodología aplicada y los atributos seleccionados para este estudio en el cuarto epígrafe. Finalmente se presentan los resultados del trabajo, así como las conclusiones derivadas del mismo.

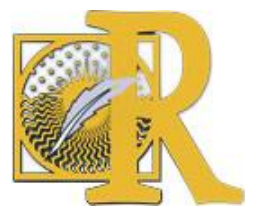

Fecha de recepción: 15-01-2020 Fecha de aceptación: 16-02-2020

Martín-Caraballo, A. M., Paralera-Morales, C. \& Ramírez-Hurtado, J. M. (2021). Estudio de la satisfacción de

los estudiantes universitarios con asignaturas de contenido cuantitativo.

International Journal of Educational Research and Innovation (IJERI), 16, 162-182

ISSN: 2386-4303 DOI https://doi.org/10.46661/ijeri.4542 


\section{Antecedentes, evolución y momento actual del espacio europeo de educación} superior.

Hablar de Europa no es sólo hablar en un espacio común de convivencia, cooperación política y económica, es también pensar en una Europa unida de los conocimientos. Fue en el año 1998 cuando los ministros responsables de la educación superior de Alemania, Francia, Italia y Reino Unido plantearon esta reflexión, que fue plasmada en la Declaración de la Sorbona, considerada como el punto de partida para el diseño de un nuevo sistema de Educación Superior Europeo (EEES) que más tarde fue conocido como "proceso de Bolonia" en 1999. En el proceso de convergencia europea son pilares claves: El Aprendizaje Centrado en el Estudiante; un Sistema de Créditos ECTS; tres ciclos formativos; el Suplemento Europeo al Título; el Establecimiento de sistemas de calidad y la Formación Basada en Competencias. Así, los roles de profesores y estudiantes cambian. El estudiante pasa a tener un rol mucho más activo y menos dependiente de lo que venía siendo tradicionalmente, situándose en el centro de la formación; mientras que el profesor asume el rol de orientador y dinamizador, es decir no es más que un mero transmisor de conocimientos. Por tanto, en este cambio de paradigma es importante tener instrumentos que nos permitan medir la satisfacción/valoración de la docencia desde el punto de vista del estudiante, que es ahora parte muy importante y activa del proceso de enseñanza-aprendizaje.

A modo de resumen, en la Figura 1 se presenta cómo quedan diseñadas las enseñanzas en el sistema universitario español, según el modelo EEES, en el que se establecen tanto las enseñanzas de primer ciclo, como de segundo y tercer ciclo.

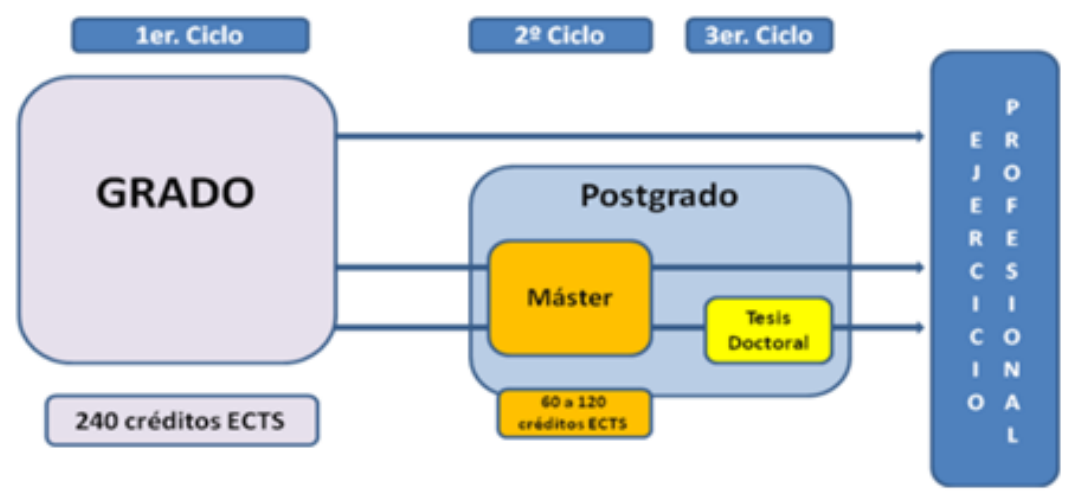

Figura 1. Estructura de la enseñanza universitaria en el marco EEES.

\subsection{La satisfacción de los estudiantes con la docencia universitaria.}

La satisfacción de los estudiantes con la docencia es uno de los aspectos más importantes en el contexto de la educación superior. Sin embargo, su estudio puede ser realizado desde diversos puntos de vista y con distintas perspectivas. Pérez et al. (2010), hacen referencia a ésta como la "satisfacción por la formación recibida". Otros autores como Oliver y DeSarbo (1989) contemplan que la satisfacción del estudiante tiene en cuenta las evaluaciones subjetivas de los resultados y experiencias relacionadas con la educación. Oliver (1980) refleja en su trabajo que la satisfacción del estudiante está constantemente influenciada por

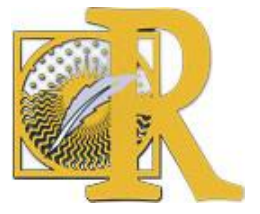


las experiencias de otros estudiantes. También ha de considerarse que lo que les ocurre a los estudiantes en el aula y sus opciones académicas no son independientes de otras experiencias en el campus. La combinación de estos factores afecta a la satisfacción del estudiante con la universidad (Seymour, 1993).

Es importante encontrar formas fiables de medir la satisfacción del alumno en la enseñanza universitaria ya que esto permitirá conocer la realidad de la misma, compararla con otras y analizarla a lo largo del tiempo, ya que el grado de satisfacción de los estudiantes por la formación docente puede llevar a la permanencia de los mismos en la universidad, a alcanzar el éxito en el desarrollo de su carrera y a transmitir su valoración a los demás, lo que hará que la institución se haga más competitiva frente a otra.

A la hora de medir la satisfacción se utilizan diferentes técnicas, desde las más tradicionales donde el nivel de satisfacción del estudiante está basado en resultados de análisis descriptivos, de importancia-resultado percibido y análisis de regresión, hasta las más actuales que utilizan modelos de ecuaciones estructurales para analizar las relaciones entre el resultado percibido, los resultados y la satisfacción en la enseñanza universitaria (Alves y Raposo, 2004).

Son muchos los autores que utilizan en sus estudios estas herramientas. Entre los que utilizan la regresión para medir el nivel de satisfacción del alumno, podemos destacar los resultados aportados por Letcher y Neves (2010). Estos autores muestran que el asesoramiento y la calidad de la enseñanza en la materia tienen poco o ningún efecto sobre la satisfacción general de los estudiantes. Los tres factores que tienen un mayor impacto en la satisfacción son la confianza en sí mismo, las actividades extra-curriculares y las oportunidades de carrera, y la calidad de la enseñanza en general (satisfacción con la docencia en la materia). En el caso de Elliott y Healy (2001) utilizan el análisis de regresión para encontrar que los factores que fueron significativos en la predicción de la satisfacción global fueron el clima del campus, la eficacia de instrucción, excelencia en el servicio y servicios de apoyo. En el mismo sentido, Lapidus y Brown (1993), analizan la experiencia universitaria en varios campos distintos, la vida en el campus, instrucciones académicas, instrucciones institucionales, apoyo a la enseñanza y guía del estudiante, usando medidas de importancia, expectativas y satisfacción.

Otros autores también utilizan en sus estudios diferentes tipos de cuestionarios. El uso del cuestionario para la recopilación de la información es una práctica muy extendida y previa al análisis de los mismos mediante las técnicas anteriormente citadas. En concreto, el cuestionario (SEUE) con el que se recopila información para conocer la satisfacción del estudiante por los resultados y experiencias asociadas con su educación. Los autores definen la satisfacción de los alumnos como "la apreciación favorable que hacen los estudiantes de los resultados y experiencias asociadas con su educación, en función de la atención a sus propias necesidades y al logro de sus expectativas" (Gento y Vivas, 2003).

Una de las consecuencias de un buen grado de satisfacción es la elección de una determinada universidad para realizar los estudios. Si los estudiantes están satisfechos con los servicios prestados y con la calidad percibida, tienen mayores razones por las que matricularse en una universidad en concreto en cursos superiores. Estos aspectos son analizados mediante entrevistas aleatorias estratificadas en Petruzzellis et al. (2006). Es por

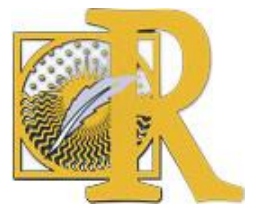


ello que las universidades deben concentrar sus esfuerzos en mejorar tanto la calidad de la docencia como la de otros servicios no relacionados con ésta.

También relacionada con el estudio de la calidad percibida, Alves y Raposo (2007) nos muestran que la variable que más influencia tiene en la satisfacción del estudiante es la imagen de la institución, seguida de la calidad percibida. Además, nos indica que hay una influencia negativa con las expectativas del estudiante. Se hace evidente que la principal consecuencia de la satisfacción es la causada por la confianza en el boca a boca de estudiante a estudiante.

En general, en los trabajos anteriormente citados no se mide exactamente el nivel de satisfacción del estudiante. En alguno de ellos se mide la calidad de la experiencia educativa o el hecho de poder recomendar la universidad en la que cursaron sus estudios a otros estudiantes. Son muchos y distintos los aspectos o atributos que se tienen en cuenta en la literatura existente sobre la valoración de la satisfacción del estudiante y la metodología empleada para mediarla, tanto atributos relacionados con la infraestructura, servicios universitarios, vida universitaria, organización académica como en la enseñanza entre otros. En definitiva, hay que señalar que el estudio de la satisfacción del alumnado universitario es muy diverso y se puede realizar desde diferentes perspectivas. La heterogeneidad de los sistemas educativos hace aconsejable la realización de estudios focalizados en materias comunes. Este trabajo contribuye a ello, analizando la satisfacción de los estudiantes universitarios con materias o asignaturas de contenidos cuantitativos.

\section{Metodología.}

\subsection{Muestra.}

El contenido se escribe aquí. Los datos de este trabajo fueron obtenidos a partir de un cuestionario dirigido a los estudiantes de asignaturas de contenido cuantitativo de varias universidades españolas. Las universidades participantes en este estudio fueron la Universidad de Extremadura, la Universidad de Granada, la Universidad de Málaga, la Universidad Pablo de Olavide de Sevilla, la Universidad Politécnica de Cataluña y la Universidad de Valencia. Los cuestionarios iban dirigidos a los alumnos matriculados en asignaturas relacionadas con métodos cuantitativos (matemáticas, estadística y econometría). Los alumnos tenían que puntuar la importancia y la valoración de una serie de atributos relacionados con la docencia, en una escala de 0 a 10 puntos, donde 0 representaba "nada importante" ("valoración más baja") y 10 representaba "muy importante" ("valoración más alta"). Se utilizó un muestreo por conveniencia, obteniendo un tamaño muestral de 344 estudiantes. Los datos fueron obtenidos a mediados del curso 2016-2017, para que los alumnos tuvieran experiencia en el recibimiento de la docencia de las asignaturas señaladas anteriormente. En la Tabla 1 aparece recogido el tamaño muestral desagregado por universidades. Se puede observar que la Universidad Pablo de Olavide tiene un tamaño muestral muy superior al resto de universidades. Esto es debido a que los autores del trabajo pertenecen a esta universidad, con lo que la obtención de datos ha sido mucho más fácil.

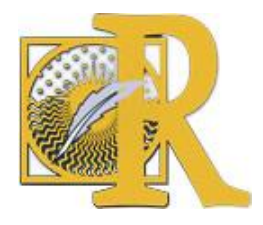

Fecha de recepción: 15-01-2020 Fecha de aceptación: 16-02-2020 
Tabla 1. Tamaño muestral desagregado por universidades.

\begin{tabular}{|c|c|}
\hline Universidad & Tamaño muestral \\
\hline Universidad de Extremadura & 13 \\
Universidad de Granada & 21 \\
Universidad de Málaga & 14 \\
Universidad Pablo de Olavide & 278 \\
Universidad Politécnica de Cataluña & 12 \\
Universidad de Valencia & 6 \\
\hline TOTAL & $\mathbf{3 4 4}$ \\
\hline
\end{tabular}

\subsection{Atributos.}

Para poder medir la satisfacción que los alumnos experimentan con materias de contenidos cuantitativos se utilizaron 18 atributos (Tabla 2). Estos atributos estaban relacionados con el desarrollo de las clases, el contenido, la evaluación y el control del profesorado. La elección de los atributos se hizo en función de la literatura existente al respecto y en función de la estructura de los estudios de las asignaturas objeto de estudio.

Tabla 2. Atributos seleccionados para la medición de la satisfacción.

Fuente: elaboración propia.

\begin{tabular}{|l|}
\hline Contenido de clases teóricas \\
\hline Contenido de clases prácticas \\
\hline Software utilizadoática en la asignatura \\
\hline Temporalización y distribución de los diversos contenidos \\
\hline Trabajo autónomo necesario para superar la asignatura \\
\hline Inclusión de materiales en el aula virtual \\
\hline Utilidad de la Guía Docente de la asignatura \\
\hline Identificación y consecución de objetivos/competencias de la asignatura \\
\hline Bibliografía proporcionada para la asignatura \\
\hline Sistema de evaluación continua \\
\hline Evaluación final \\
\hline Evaluación virtual \\
\hline Tutorías presenciales \\
\hline Tutorías virtuales \\
\hline Comunicación virtual profesor-alumno \\
\hline Control de seguimiento-asistencia por el profesor \\
\hline Acceso a calificaciones por parte del alumno \\
\hline
\end{tabular}

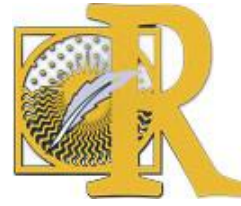


El análisis de la satisfacción de los estudiantes universitarios con estos 18 atributos permite identificar lagunas donde la experiencia de los estudiantes no está en consonancia con las expectativas esperadas. Por tanto, se podrían identificar los atributos que provocan una mayor satisfacción en el alumnado y los que provocan una mayor insatisfacción. En este último caso, esta información sería de una utilidad muy valiosa ya que se trataría de posibles defectos en la docencia que podrían ser corregidos con el objetivo de incrementar la calidad docente.

\subsection{El análisis de importancia-valoración (IPA).}

Para conseguir el objetivo de este trabajo se ha utilizado una variante del Análisis de Importancia-Valoración (IPA: Importance-Performance Analysis) de Martilla y James (1977) propuesta por algunos autores (Ábalo et al., 2006; Picón et al., 2011). El IPA es una de las técnicas más útiles para desarrollar acciones de marketing ya que permite representar de manera sencilla y funcional los puntos fuertes y áreas de mejora de un determinado producto o servicio (Ábalo et al., 2006). Esta herramienta consiste básicamente en analizar conjuntamente tanto la importancia que los consumidores y usuarios conceden a los atributos que componen un producto o servicio, como la valoración que hacen de los mismos tras su utilización y disfrute (Picón et al., 2011).

A pesar de que esta técnica tiene su origen en el área de marketing, también ha sido aplicada en campos muy diversos como el sanitario, educativo, industrial, deportivo o turístico.

Destacar de nuevo que en el IPA los individuos tienen en cuenta la importancia que conceden a los distintos atributos de un producto o servicio, así como la valoración que hacen de los mismos una vez que ya han experimentado el producto o servicio.

Los tradicionales modelos multiatributos y de expectativa-valor sostienen que cada producto o servicio está compuesto por una serie de atributos independientes, de modo que las valoraciones de los consumidores se construyen a partir de la agregación ponderada de las evaluaciones de cada uno de estos atributos (Fishbein, 1967). Todo esto quedaría reflejado en la siguiente expresión (Fishbein y Ajzen, 1975):

$$
V_{0}=\sum_{i=1}^{n} I_{i} \cdot V_{i}
$$

Donde $V_{0}$ es la valoración global del producto o servicio, $I_{i}$ es la importancia que tiene cada atributo para los sujetos, $V_{i}$ es la valoración que recibe cada atributo y $n$ es el número total de atributos que configuran el producto o servicio.

La literatura ha puesto de manifiesto que no todos los atributos explican por igual la satisfacción global con un producto o servicio (Picón et al., 2011). Los consumidores suelen hacer las valoraciones basándose en un número limitado de características, de modo que los atributos más importantes afectan en gran medida a la evaluación del usuario, mientras que los menos importantes apenas influyen. Por ello, ponderar las valoraciones de los diferentes atributos es un aspecto que adquiere una gran relevancia.

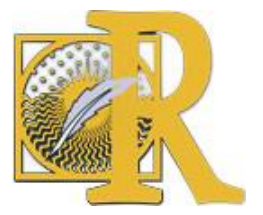


A diferencia de los modelos multiatributos de Fishbein (1967), en los que la importancia y la valoración se analizan de forma agregada, en el IPA la importancia y la valoración se analizan de forma separada. Esto es importante porque una puntuación alta puede obtenerse mediante una alta importancia y baja valoración o mediante una baja importancia y alta valoración (Ennew et al., 1993).

La representación clásica del IPA propuesto por Martilla y James (1977) es en dos ejes que definen cuatro cuadrantes (Figura 2). En el eje de ordenadas se representan las Importancias, mientras que en el eje de abscisas se representan las Valoraciones. Los puntos que se representan en el gráfico corresponden a las puntuaciones medias de la importancia y de la valoración, obtenidas para cada atributo. Los cuatro cuadrantes están caracterizados como se aprecia en la Figura 2.

A. Concentrarse aquí - alta importancia, baja valoración;

B. Mantener el buen trabajo -alta importancia, alta valoración;

C. Baja prioridad - baja importancia, baja valoración;

D. Posible derroche de recursos - baja importancia, alta valoración.

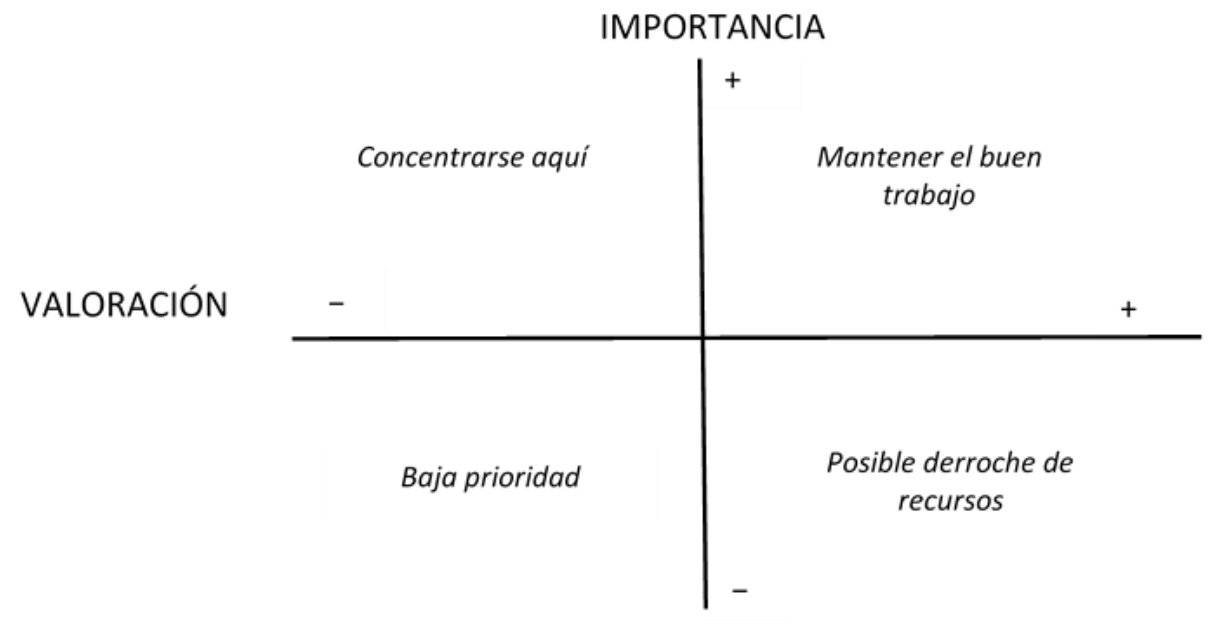

Figura 2. Representación clásica del Análisis de Importancia-Valoración.

Fuente: elaboración propia a partir de Martilla y James (1977).

La prioridad para la empresa o institución debe ser el cuadrante A, es decir, atender con mayor rapidez los atributos que caigan en este cuadrante. Así pues, en función del cuadrante sobre el que caiga cada uno de los atributos, el responsable de marketing (en este caso de los responsables de las asignaturas del estudio) deberá definir la estrategia a seguir. La metodología, aunque se ha aplicado originariamente en el área de marketing, se ha venido aplicando en distintos campos: el sanitario, educativo, industrial, marketing interno, calidad de servicio o deportivo. La técnica se ha aplicado también, más recientemente, en calidad de servicios (Gil et al., 2016; Martínez-Caro et al., 2016; Zamorano y García, 2018), en la gestión relacionada con el deporte (Martínez, 2018; Serrano et al., 2014; Serrano-Gómez et al., 2013)

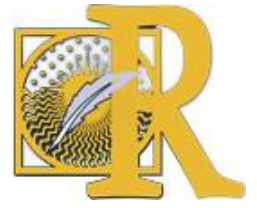


o en la elección de herramientas tecnológicas aplicadas en los procesos de aprendizaje (Ortigueira, 2016).

En concreto, en el campo educativo hay que resaltar los trabajos de Alberty y Mihalik (1989), Ortinau et al. (1989), Ford et al. (1999), Nale et al. (2000) y Pike y Larkin (2010). Alberty y Mihalik (1989) aplicaron el análisis de importancia-valoración en la educación de adultos. Ortinau et al (1989) mostraron en su estudio cómo aplicar el análisis de importanciavaloración en la educación. Para ello evaluaron una serie de atributos correspondientes a un curso universitario. Por su parte, Ford et al. (1999) utilizaron el análisis de importanciavaloración para identificar las percepciones de estudiantes universitarios sobre la calidad de una serie de factores que deben poseer las universidades. Más tarde, Nale et al. (2000) estudiaron las percepciones de una serie de estudiantes de una escuela de negocio, acerca de cómo habían estado formados para las carreras que habían elegido. Finalmente, Pike y Larkin (2010) utilizan el análisis de importancia-valoración para poder evaluar la satisfacción de los estudiantes con un programa de postgrado. Los resultados mostraron que varios factores analizados debían ser corregidos.

A pesar de ser una técnica que ofrece de manera sencilla y directa implicaciones para posteriores acciones de marketing y a pesar de ser aplicada en ámbitos muy diversos, sin embargo, también presenta algunos puntos débiles, como pueden ser la colocación de los ejes o la medición de la importancia de los atributos (Picón et al., 2011). Para evitar estos problemas, Picón et al. (2011) proponen una nueva forma de representar gráficamente los resultados teniendo en cuenta el análisis de las discrepancias, es decir, las diferencias entre las importancias y las valoraciones. Con ello se pretende solucionar el problema de la arbitrariedad que existe a la hora de posicionar los ejes, así como incrementar la cantidad de información que proporciona la representación de los cuadrantes. Este estudio sigue esta metodología propuesta por Picón et al. (2011).

\section{Resultados.}

En la Tabla 3 vienen recogidas las puntuaciones medias de la importancia y valoración de cada uno de los 18 atributos. Un primer resultado importante es que la media total de la importancia $(7,12)$ es superior a la media de la valoración $(6,91)$, por lo que, en principio esto muestra un cierto grado de insatisfacción en los estudiantes. Si se analizan detenidamente las diferencias entre las puntuaciones medias de la valoración y las puntuaciones medias de la importancia, obtenemos lo que se denominan discrepancias. Las discrepancias negativas informan de aquellos atributos que son susceptibles de ser mejorados, ya que la puntuación en la valoración es inferior a la puntuación en la importancia.

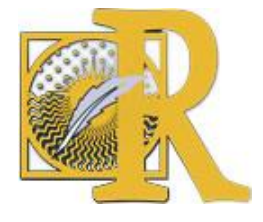

Fecha de recepción: 15-01-2020 Fecha de aceptación: 16-02-2020 
Así pues, se puede observar que todos los atributos presentan discrepancias negativas salvo el atributo de "Trabajo autónomo necesario para superar la asignatura", el cual presenta una discrepancia positiva, lo que significa que los estudiantes están satisfechos con este factor.

Tabla 3. Puntuaciones medias de la valoración e importancia de los atributos.

\begin{tabular}{|l|l|c|c|c|}
\hline & Valoración & Importancia & Discrepancias \\
\hline 1 & Contenido de clases teóricas & 6,86 & 6,94 & $-0,08$ \\
\hline 2 & Contenido de clases prácticas & 8,36 & 8,83 & $-0,47$ \\
\hline 3 & Clases de informática & 6,79 & 7,25 & $-0,46$ \\
\hline 4 & Software utilizado en la asignatura diversos & 6,50 & 6,62 & $-0,12$ \\
\hline & Temporalización y distribución de los didos & 6,78 & 7,07 & $-0,29$ \\
\hline 6 & contenidos & 7,94 & 7,86 & 0,08 \\
\hline 7 & Irabajo autónomo necesario para superar la asignatura & 7,55 & 7,61 & $-0,06$ \\
\hline 8 & Utilidad de la Guía Docente de la asignatura & 6,07 & 6,22 & $-0,14$ \\
\hline & Identificación y consecución de objetivos/competencias & & & \\
9 & de la asignatura & 6,76 & 6,89 & $-0,13$ \\
\hline 10 & Bibliografía proporcionada para la asignatura & 5,87 & 6,04 & $-0,18$ \\
\hline 11 & Sistema de evaluación continua & 7,43 & 7,56 & $-0,13$ \\
\hline 12 & Evaluación final & 7,60 & 7,83 & $-0,23$ \\
\hline 13 & Evaluación virtual & 6,04 & 6,22 & $-0,18$ \\
\hline 14 & Tutorías presenciales & 7,22 & 7,41 & $-0,20$ \\
\hline 15 & Tutorías virtuales & 5,43 & 5,69 & $-0,26$ \\
\hline 16 & Comunicación virtual profesor-alumno & 6,80 & 7,03 & $-0,23$ \\
\hline 17 & Control de seguimiento-asistencia por el profesor & 6,40 & 6,56 & $-0,16$ \\
\hline 18 & Acceso rápido a calificaciones por parte del alumno & 7,97 & 8,48 & $-0,50$ \\
\hline & Media & 6,91 & 7,12 & \\
\hline
\end{tabular}

Fuente: elaboración propia.

La Figura 3 recoge la representación gráfica clásica de Martilla y James (1977). Se puede observar que todos los atributos caen en el cuadrante de "mantener el buen trabajo", sin embargo, se ha visto previamente que casi todos los atributos son susceptibles de ser mejorados. Por tanto, esta representación gráfica no refleja la realidad, ya que los atributos no presentarían deficiencias. Tal como señalan Ábalo et al. (2006), es necesario plantear una modificación del gráfico para que la representación sea más coherente y acorde a la realidad. 


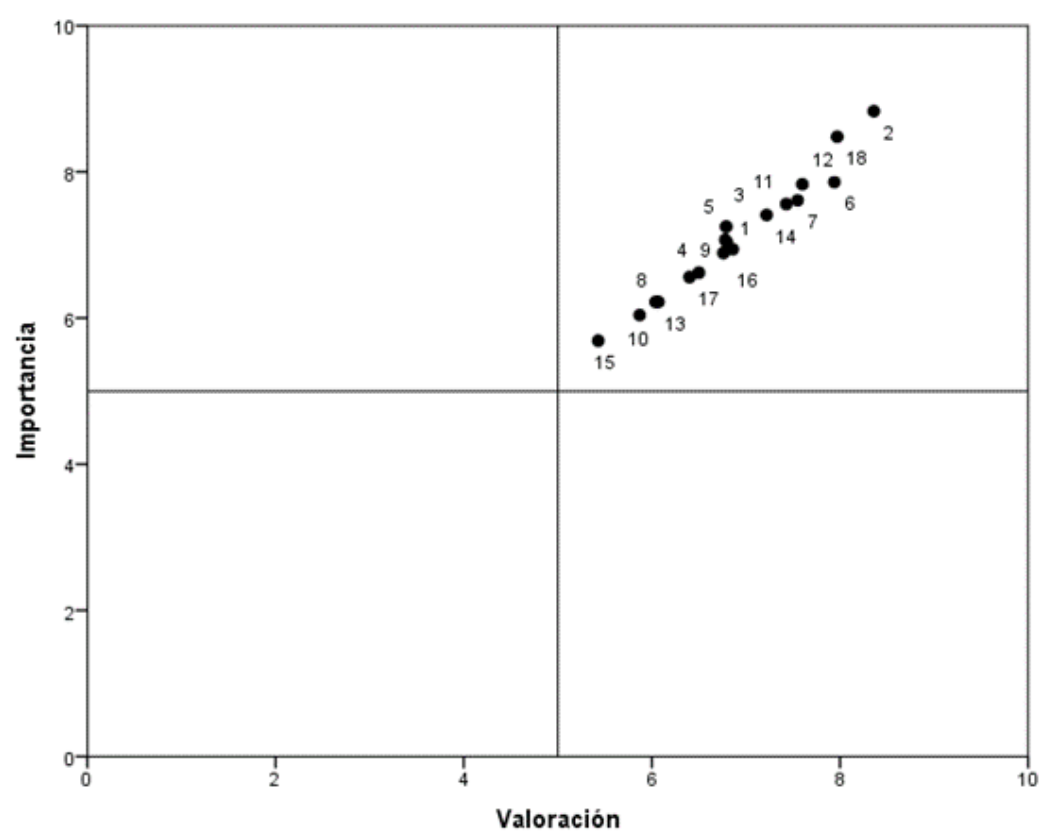

Figura 3. Representación clásica de Martilla y James (1977).

Fuente: elaboración propia.

Según Ábalo et al. (2006), una primera transformación, ya apuntada por Martilla y James (1977), consiste en modificar la escala de los ejes. Estos autores proponen situar el inicio de los ejes según el valor más pequeño obtenido en las puntuaciones medias. En diversos estudios de estos autores el final de cada eje no se ha modificado. Con el fin de hacer una transformación coherente y equitativa, en este estudio proponemos también modificar el final de los ejes según el valor más alto obtenido. Así pues, la nueva representación gráfica tomaría como inicio de los ejes el valor 5 y como punto final de los ejes el valor 9. Consecuentemente, el punto medio de la nueva escala será 7 .

En la Figura 4 viene recogida la nueva representación gráfica y se puede observar que casi todos los atributos caen en el cuadrante "mantener el buen trabajo" o en el cuadrante "baja prioridad". Tan sólo los atributo 3, 5 y 16 caen en el cuadrante "concentrarse aquí". Por tanto, la nueva representación sigue contando con un sesgo elevado, ya que apenas mejora la situación, debido a que sigue habiendo muchos atributos en el cuadrante "Mantener el buen trabajo" y en el cuadrante "baja prioridad", pero tan sólo hay tres atributos en el cuadrante "concentrarse aquí". Hay que recordar que las discrepancias indicaban que todos los atributos menos uno era susceptible de ser mejorados.

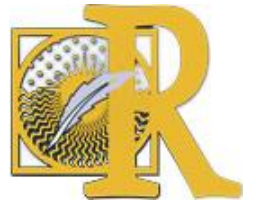




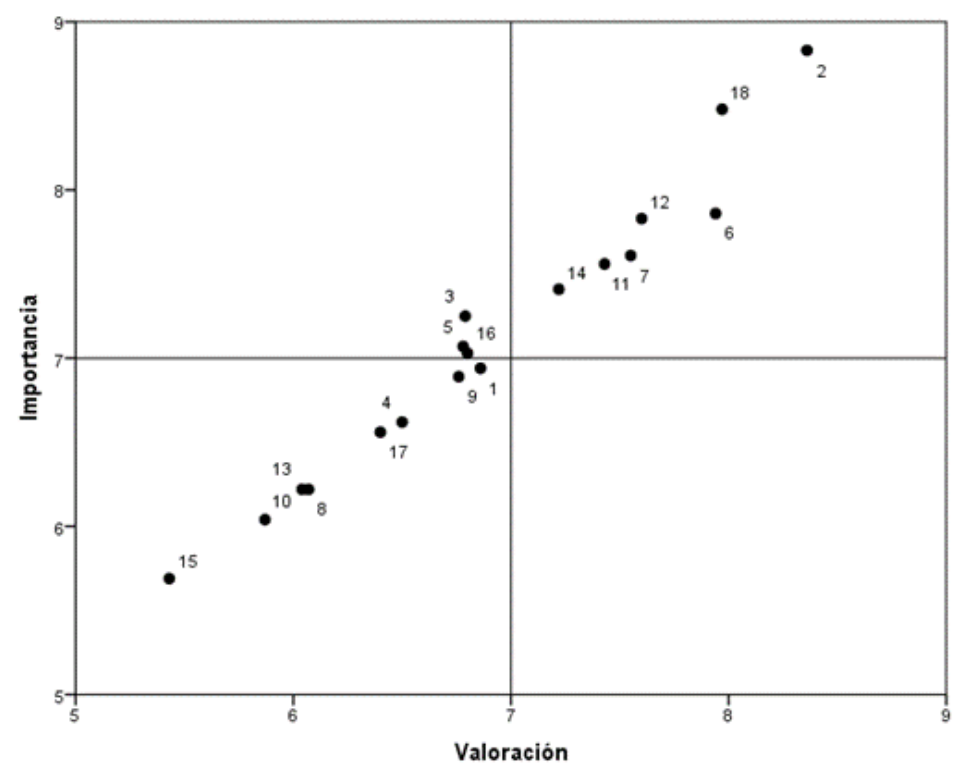

Figura 4. Representación clásica con la nueva escala.

Fuente: elaboración propia.

Según Ábalo et al. (2006), otro criterio que ha sido seguido en la literatura por algunos autores (Alberty y Mihalik, 1989; Guadagnolo, 1985; Hollenhorst et al., 1992) consiste en situar en los ejes las medias de sus respectivas dimensiones. En este caso, se obtiene una representación más o menos equitativa de los atributos entre los cuatro cuadrantes (Figura 5).

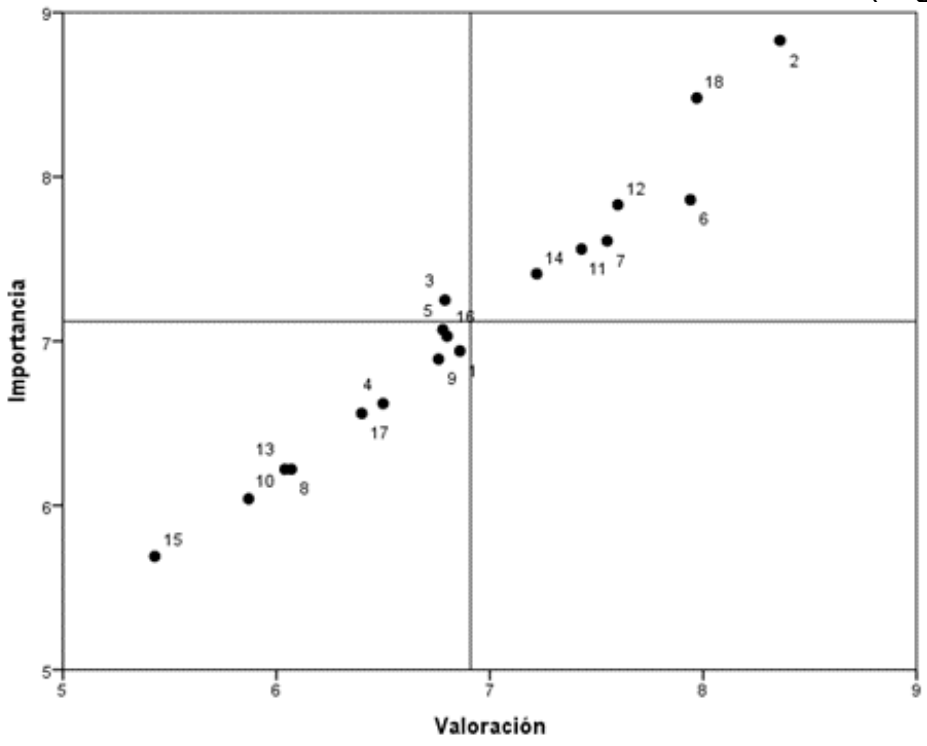

Figura 5. Representación con los ejes en la media de la Valoración $(6,91)$ y de la Importancia $(7,12)$. Fuente: elaboración propia.

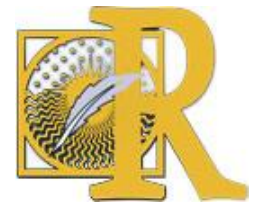


Sin embargo, a pesar de que esta representación distribuye de forma más o menos equitativa los puntos entre los cuatro cuadrantes, se considera que es una forma parcialmente artificiosa de los puntos fuertes y de las áreas de mejora (Ábalo et al., 2006). De hecho, en el caso que se está estudiando, el único atributo que cae en el cuadrante "concentrarse aquí" es el atributo 3. Por tanto, esta representación gráfica sigue sin reflejar la realidad existente.

Para solventar todos los problemas anteriores, la literatura ha tratado de combinar los modelos basados en la representación en cuadrantes (modelos clásicos) frente a los denominados "modelos diagonales", que dividen el espacio del análisis de importanciavaloración en dos mitades (Ábalo et al., 2006; Hawes y Rao, 1985; Nale et al., 2000; Picón et al., 2011; Sampson y Showalter, 1999; Slack, 1994).

Los modelos diagonales están basados en el cálculo de las discrepancias, definidas como las diferencias en las puntuaciones entre las valoraciones y las importancias. Aquellos atributos con bajas discrepancias son aquellos donde la importancia excede a la valoración, por lo que representan altas prioridades para la mejora. En este tipo de modelos se representa la diagonal de $45^{\circ}$ en el gráfico, de modo que aquellos puntos situados por encima de la diagonal tienen discrepancias negativas y, por tanto, son susceptibles de mejora. Mientras mayor sea la distancia del punto a la diagonal mayor será su prioridad para concentrarse en él.

En la Figura 6 aparecen combinados los modelos clásicos frente a los modelos diagonales.

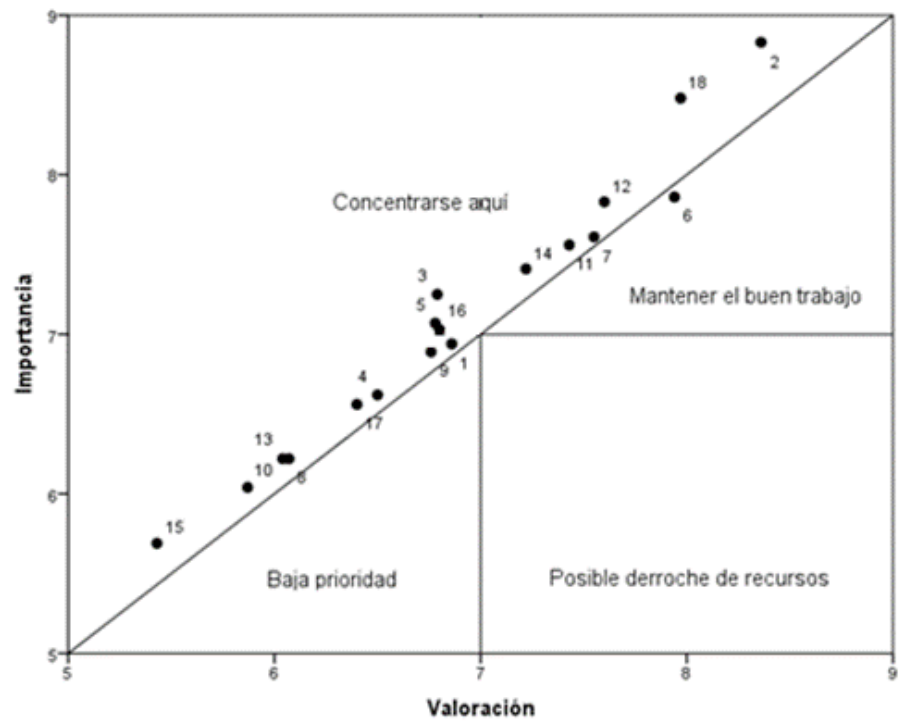

Figura 6. Representación combinada de modelos clásicos y modelos diagonales.

Fuente: elaboración propia.

Lo primero que se puede deducir del gráfico es que ahora todos los puntos caen por encima de la diagonal, salvo el atributo 6, con lo cual los atributos son susceptibles de ser mejorados. Estos atributos se corresponden con aquellos cuyas discrepancias son negativas. Aquellos atributos más alejados de la diagonal son aquellos cuya prioridad es más elevada, debido a

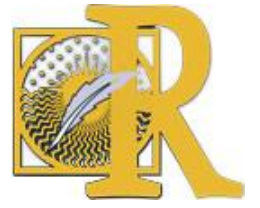


que la diferencia entre la importancia y la valoración es más elevada. Se puede observar que los atributos más alejados de la diagonal y que caen en el triángulo superior son el 18 (acceso rápido a calificaciones por parte del estudiante), 2 (contenido de las clases prácticas) y 3 (clases de informática).

También se puede observar en el gráfico que tan sólo hay un punto que destaca por estar por debajo de la diagonal, el cual se corresponde con el atributo 6 (trabajo autónomo necesario para superar la asignatura), el cual cae en el triángulo de "mantener el buen trabajo". Otro aspecto destacable es que no hay puntos en el triángulo de "baja prioridad" y en el cuadrante de "posible derroche de recursos".

El resto de atributos se encuentran por encima de la diagonal, lo que señala que son susceptibles de ser mejorados, pero no con tanta prioridad como los mencionados inicialmente. En resumen, se puede indicar que los estudiantes muestran insatisfacción con la docencia de asignaturas de tipo cuantitativo.

Para estudiar si hay diferencias significativas por universidades se ha comprobado en primer lugar si los datos siguen una distribución normal mediante el contraste de KolmogorovSmirnov y el contraste de Shapiro-Wilks. Los resultados muestran que no todas las muestras siguen una distribución normal, por lo que para poder comprobar si hay diferencias significativas por universidades se ha utilizado un contraste no paramétrico, como es el test de Kruskal-Wallis. Los resultados obtenidos tanto para la importancia como para la valoración de los atributos vienen recogidos en las tablas 4 y 5 . Como se puede observar hay algunos atributos en los que hay diferencias significativas por universidades, en relación a la puntuación que los estudiantes otorgan tanto a la importancia como a la valoración. La tabla 6 recoge un resumen de estas diferencias.

\begin{tabular}{|c|c|c|c|c|c|c|c|c|c|c|c|c|c|c|c|c|c|c|}
\hline \multicolumn{19}{|c|}{ Estadisticos de prueba ${ }^{\mathrm{a}, \mathrm{b}}$} \\
\hline & $\pi$ & 12 & 13 & 14 & 15 & 16 & 17 & 18 & 19 & 110 & I11 & 1112 & $\overline{113}$ & 114 & 115 & 116 & 1177 & 118 \\
\hline Chi-cuadrado & 4,373 & 7,477 & 18,543 & 27,025 & 23,071 & 8,295 & 3,761 & 10,080 & 14,142 & 8,166 & 6,454 & 13,935 & 4,774 & 9,807 & 5,362 & 4,101 & 14,218 & 9,413 \\
\hline او او او & 5 & 5 & 5 & 5 & 5 & 5 & 5 & 5 & 5 & 5 & 5 & 5 & 5 & 5 & 5 & 5 & 5 & 5 \\
\hline Sig asintiotca & .497 & .187 & .002 & .000 & .000 & .141 & .584 & .073 & .015 & .147 & .264 & .016 & .444 & .081 & .373 & .535 & .014 & .094 \\
\hline
\end{tabular}

a. Prueba de Kruskal Wallis

b. Variable de agrupación: Universidad

\begin{tabular}{|c|c|c|c|c|c|c|c|c|c|c|c|c|c|c|c|c|c|c|}
\hline \multicolumn{19}{|c|}{ Estadisticos de prueba ${ }^{a, b}$} \\
\hline & Vi & $\sqrt{2}$ & $\sqrt{3}$ & $\sqrt{14}$ & V5 & V6 & $\sqrt{7}$ & $\sqrt{8}$ & $\sqrt{9}$ & V10 & V11 & $\mathrm{V} 12$ & $\mathrm{~V} 13$ & V14 & V15 & $\mathrm{V} 16$ & V17 & V18 \\
\hline Chi-cuadrado & 3,643 & 31,186 & 24,378 & 16,209 & 3,452 & 8,898 & 23,102 & 2,977 & 4,994 & 3,516 & 3,569 & 6,194 & 16,850 & 13,837 & 11,998 & 10,491 & 10,643 & 23,360 \\
\hline او & 5 & 5 & 5 & 5 & 5 & 5 & 5 & 5 & 5 & 5 & 5 & 5 & 5 & 5 & 5 & 5 & 5 & 5 \\
\hline Sig. asintiotca & .602 & .000 & .000 & .006 & .631 & .113 & .000 & .704 &, 417 & .621 & .613 & .288 & .005 & .017 & .035 & .062 & .059 & .000 \\
\hline
\end{tabular}

a. Prueba de Kruskal Wallis

b. Variable de agrupación: Univetsidad

Tabla 6. Diferencias significativas en la Importancia y Valoración por universidades.

\begin{tabular}{|c|c|c|c|}
\hline & $x_{1}$ & Importancia & Valoración \\
\hline 1 & Contenido de clases teóricas & No & No \\
\hline 2 & Contenido de clases prácticas & No & Sí \\
\hline 3 & Clases de informática & Sí & Sí \\
\hline 4 & Software utilizado en la asignatura & Sí & Sí \\
\hline 5 & Temporalización y distribución de los diversos contenidos & Sí & No \\
\hline
\end{tabular}

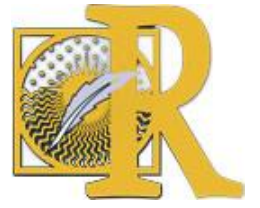




\begin{tabular}{|c|c|c|c|}
\hline 6 & Trabajo autónomo necesario para superar la asignatura & No & No \\
\hline 7 & Inclusión de materiales en el aula virtual & No & Sí \\
\hline 8 & Utilidad de la Guía Docente de la asignatura & No & No \\
\hline 9 & Identificación y consecución de objetivos/competencias de la asignatura & Sí & No \\
\hline 10 & Bibliografía proporcionada para la asignatura & No & No \\
\hline 11 & Sistema de evaluación continua & No & No \\
\hline 12 & Evaluación final & Sí & No \\
\hline 13 & Evaluación virtual & No & Sí \\
\hline 14 & Tutorías presenciales & No & Sí \\
\hline 15 & Tutorías virtuales & No & Sí \\
\hline 16 & Comunicación virtual profesor-alumno & No & No \\
\hline 17 & Control de seguimiento-asistencia por el profesor & Sí & No \\
\hline 18 & Acceso rápido a calificaciones por parte del alumno & No & Sí \\
\hline
\end{tabular}

Fuente: elaboración propia.

Cabe destacar que tanto en las clases de informática como en el software utilizado en la asignatura hay diferencias significativas en la importancia y en la valoración que los estudiantes de las distintas universidades conceden a estos dos atributos. En el resto de atributos las diferencias se encuentran o bien en la importancia o bien en la valoración. En definitiva, los resultados muestran que hay diferencias significativas en la forma en que los estudiantes perciben y reciben la docencia de asignaturas de contenido cuantitativo en las distintas universidades.

\section{Conclusiones.}

El entorno dinámico y competitivo que envuelve al sector educativo exige una orientación al mercado para que la educación y el aprendizaje sean exitosos. Para poder desarrollar estrategias que permitan alcanzar niveles de calidad en los centros educativos, los administradores y gestores deben identificar las fortalezas y debilidades de los sistemas educativos. Sin lugar a dudas, este tipo de información puede resultar de gran utilidad para poder implementar estrategias en aras de mejorar la calidad de los sistemas.

La docencia recibida por estudiantes universitarios y del aprendizaje llevado a cabo por los mismos depende de una gran cantidad de factores y criterios, como pueden ser el material, el desarrollo de las clases teóricas, la impartición de clases prácticas, las tutorías, la forma de evaluación, etc. Para poder alcanzar niveles elevados de calidad en la docencia y en el aprendizaje es necesario identificar cuáles de estos factores se desarrollan de forma correcta y cuáles deben ser corregidos por tener una implementación incorrecta.

En el caso concreto de este estudio, los resultados muestran qué factores o atributos proporcionan una mayor satisfacción a los estudiantes universitarios de materias cuantitativas y cuáles son los que provocan una mayor insatisfacción. Esta información permite recoger qué factores tienen un mal desempeño y, por tanto, deben ser corregidos para mejorar la calidad de la docencia de materias cuantitativas. Esta información puede resultar muy valiosa para que muchas instituciones universitarias puedan orientar la docencia de estas materias con el fin de incrementar la satisfacción del alumnado.

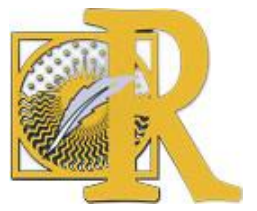


Como se puede observar, los datos de este estudio están obtenidos desde el punto de vista de los estudiantes universitarios. Hay que tener presente que las percepciones de los estudiantes sobre la docencia recibida y el aprendizaje alcanzado son consideradas importantes debido fundamentalmente a dos razones. Por un lado, los estudiantes universitarios no son únicamente "productos" de la enseñanza superior, sino que además son clientes que tratan de satisfacer sus necesidades. Por otro lado, dentro del sistema educativo, los estudiantes universitarios se encuentran en una excelente posición para poder detectar las fortalezas y debilidades de la formación que reciben. Así pues, estos dos hechos justifican la importancia de la información obtenida de las percepciones de los estudiantes universitarios.

Los resultados de este trabajo muestran que los atributos que caracterizan a la docencia en asignaturas de contenido cuantitativo caen muy cercanos a la diagonal del modelo de importancia-valoración. Esto significa que, por un lado, los alumnos muestran un nivel moderado de satisfacción con la docencia de estas asignaturas. Es decir, no hay atributos que reflejen un alto grado de satisfacción, pero tampoco hay atributos sobre los que los alumnos muestren un elevado grado de insatisfacción. Además, esto es algo generalizado para todas las universidades que han formado parte del estudio.

El análisis de las discrepancias permite deducir que los atributos que provocan una mayor insatisfacción en los estudiantes son el acceso rápido a calificaciones por parte del estudiante, el contenido de las clases prácticas, las clases de informática y la temporalización y distribución de los diversos contenidos. Por tanto, estos atributos deben ser modificados y gestionados de forma diferente para tratar de producir una mayor satisfacción en el alumnado. En el caso de las calificaciones, el profesorado debería tener presente este resultado, de modo que las pruebas de evaluación deberían ser corregidas con mayor rapidez. En algunos casos, como son los tests, podría de gran utilidad el uso de dispositivos lectores de plantillas de respuestas para correcciones automáticas, lo que reduciría el tiempo de corrección. En el caso de exámenes de tipo convencional sería conveniente que el coordinador de la asignatura haga una programación con los profesores de la misma para corregir con la mayor rapidez posible.

El sistema de evaluación final está basado en los resultados alcanzados por los alumnos en la prueba final, consistente habitualmente en un examen. Para el alumnado la medición de su rendimiento está basada en gran medida en una prueba final, lo cual supone para ellos un aspecto de capital importancia por el peso que tiene esta prueba. Por parte del docente existe una gran dificultad en la configuración de un instrumento de comprobación del rendimiento académico. Cada vez es más común que el peso de la prueba final sea igual o similar al de la evaluación continua, este cambio hay que hacérselo notar al alumno y que éste perciba la importancia de la evaluación continua frente a una prueba final. Por tanto, este sistema debe ser analizado de forma cuidadosa con tal de incrementar la satisfacción de los alumnos de asignaturas con contenido cuantitativo. En relación con el acceso a las calificaciones por parte de los alumnos hay que decir que éste se realiza habitualmente en estos últimos años en muchas universidades a través del aula virtual. Posiblemente este resultado pueda ser debido a que este atributo esté influenciado por el tiempo en publicar las calificaciones y por

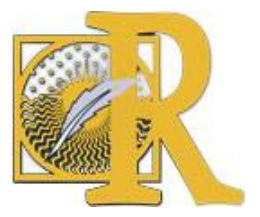

Fecha de recepción: 15-01-2020 Fecha de aceptación: 16-02-2020

Martín-Caraballo, A. M., Paralera-Morales, C. \& Ramírez-Hurtado, J. M. (2021). Estudio de la satisfacción de

los estudiantes universitarios con asignaturas de contenido cuantitativo.

International Journal of Educational Research and Innovation (IJERI), 16, 162-182

ISSN: 2386-4303 DOI https://doi.org/10.46661/ijeri.4542 
la calificación obtenida. De todos modos, los resultados muestran que el proceso para acceder a las calificaciones debe ser agilizado.

Las clases prácticas son de gran importancia para que el alumno muestre la aplicación de los conceptos teóricos adquiridos y para que mejore el aprendizaje de las diversas competencias. Sin embargo, en este caso los resultados muestran que existe una deficiencia en este atributo. Esta situación exige llevar a cabo acciones dirigidas a la mejora del desarrollo de las clases prácticas, intentando motivar al alumno para hacerle más partícipe y mejorar de esta forma el desempeño de las mismas. Asimismo, hay que intentar hacer ver al alumno, y que además lo comprenda, la importancia que tiene el trabajo autónomo por su parte con anterioridad a este tipo de enseñanza, es decir, a las clases prácticas.

En relación con el contenido de las clases de informática, sería conveniente identificar posibles carencias en la enseñanza. Además, sería conveniente estudiar qué software puede resultar más útil en la enseñanza a los estudiantes de cara a facilitar el acceso al mercado laboral.

Los resultados también muestran que es necesario que los programas de las asignaturas de contenido cuantitativo sean revisados. Con la entrada del EEES, muchas asignaturas con una duración anual pasaron a semestral. En la mayoría de los casos, el contenido anual ha sido reducido para ser explicado en una asignatura semestral, lo que lleva a una saturación de contenidos y a una incorrecta distribución de los contenidos. Así pues, es imprescindible hacer una revisión de los programas de las asignaturas eliminando o reduciendo aquellos contenidos innecesarios. Para ello también es necesario una mayor coordinación con los contenidos de otras asignaturas de la misma titulación.

Los atributos que caen en la zona de concentrarse aquí, pero están más cercanos de la diagonal son aquellos en los que el nivel de insatisfacción es mínimo. En este caso, los dos atributos que cumplen esta condición son la inclusión de materiales en el aula virtual, el contenido de las clases teóricas y el software utilizado en la asignatura. El acceso a plataforma virtuales es un aspecto destacable por los estudiantes a la hora de recibir la docencia.

Asimismo, los alumnos de asignaturas de contenido cuantitativo no dan mucha importancia ni valoran de forma elevada el software de tales asignaturas. Esto puede ser debido a que hoy día existe una gran variedad de programas muy versátiles que pueden realizar la mayoría de las operaciones que habitualmente se ejecutan en estas materias. Pero los docentes debemos también intentar utilizar diferentes tipos de software en nuestras clases teóricas para intentar ser más claros en las explicaciones teóricas así como para facilitar la "visualización" de la teoría por parte del alumnado.

Por otro lado, hay un atributo que cae en el cuadrante de mantener el buen trabajo, como es el trabajo autónomo para superar la asignatura. Sin lugar a dudas, este aspecto permite al alumno trabajar de forma autónoma y al ritmo que desee con cierta facilidad. Por tanto, este resultado muestra que la docencia debe tender hacia un mayor proceso de autonomía del alumno.

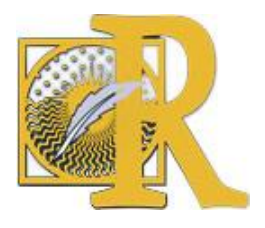

Fecha de recepción: 15-01-2020 Fecha de aceptación: 16-02-2020

Martín-Caraballo, A. M., Paralera-Morales, C. \& Ramírez-Hurtado, J. M. (2021). Estudio de la satisfacción de

los estudiantes universitarios con asignaturas de contenido cuantitativo.

International Journal of Educational Research and Innovation (IJERI), 16, 162-182

ISSN: 2386-4303 DOI https://doi.org/10.46661/ijeri.4542 
Finalmente, hay que destacar que no hay atributos que caen en el cuadrante de baja prioridad y en el cuadrante de posible derroche de recursos, lo que indica que no se están derrochando recursos en aspectos poco importantes.

En resumen, los resultados muestran en general un mínimo grado de insatisfacción con muchos atributos, aunque en muchas ocasiones este grado es casi imperceptible.

Hay que señalar que los resultados de este trabajo están condicionados de manera notable porque la muestra de estudiantes, en su mayoría, corresponde a la universidad a la que pertenecen los autores del trabajo, teniendo una baja representación del resto de universidades consideradas en el estudio. Por tanto, sería mucho más enriquecedor poder replicar este trabajo, ampliando los elementos de las muestras de otras universidades y comparando de nuevo los resultados obtenidos.

\section{Referencias.}

- Ábalo, J., Varela, J. y Rial A. (2006). El análisis de Importancia-Valoración aplicado a la gestión de servicios. Psicothema, 18, 730-737.

- Alberty, S. y Mihalik, B. J. (1989). The use of importance-performance analysis as an evaluative technique in adult education. Evaluation Review, 13(1), 33-44.

- Alves, H. y Raposo, M. (2004). La medición de la satisfacción en la enseñanza universitaria: El ejemplo de la Universidade da Beira Interior. Revista Internacional de Marketing Público y No Lucrativo, 1(1), 73-88.

- Alves, H. y Raposo, M. (2007). Conceptual model of student satisfaction in higher education. Total Quality Management, 18(5), 571-588.

- Elliot, K. M. y Healy, M. A. (2001). Key factors influencing student satisfaction related to recruitment retention. Journal of Marketing for Higher Education, 10(4), 1-11.

- Ennew, C.T., Reed, G.V. y Binks, M.R. (1993). Importance-performance analysis and the measurement of service quality. European Journal of Marketing, 27(2), 59-70.

- Escorcia, R. E., Gutiérrez, A. V., y Henríquez, H. J. (2007). La educación superior frente a las tendencias sociales del contexto. Educación y Educadores, 10(1), 63-77.

- ESU (2010). Student centered learning: Time for a new paradigm in education. European Students' Union.

- Fishbein, M.A. (1967). Attitude and the prediction of behavior, in M. Fishbein (Ed.). Readings in attitude theory and measurement. New York: Wiley, 477-492.

- Fishbein, M. A. y Ajzen, I. (1975). Belief, attitude, intention and behavior. An introduction to theory and research. Reading, Mass: Addison-Wesley.

- Ford, J.B., Joseph, M. y Joseph, B. (1999). Importance-performance analysis as a strategic tool for service marketers: The case of service quality perceptions of business students in New Zealand and the USA. Journal of Services Marketing, 13(2), 171-186.

- Froment, E., Kolher, J., Purser, L., Wilso, L., Davies, H., y Schurings, G. (2010). EUA Bologna Handbook. Making Bologna Work. Berlín, Alemania: EUA/RAABE.

- Gento, S. y Vivas, M. (2003). EI SEUE: un instrumento para conocer la satisfacción de los estudiantes universitarios con su educación. Acción Pedagógica, 12(2), 16-26.

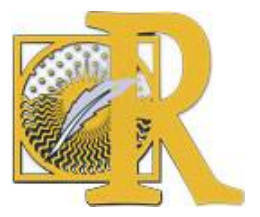


- Gil, L., Carrasco, A. y Muñoz, V.M. (2016). Evaluación de la calidad de un servicio de voluntariado en oncología un análisis importancia-valoración (IPA). Psicooncología: investigación y clínica biopsicosocial en oncología, 13(1), 71-83.

- Guadagnolo, F. (1985). The importance-performance analysis: an evaluation and marketing tool. Journal of Park Recreation Administration, 3(2), 13-22.

- Hawes, J.M. y Rao, C.P. (1985). Using Importance-Performance Analysis to develop health care marketing strategies. Journal of Health Care Marketing, 5(4), 19-25.

- Herrera, L., Lorenzo, O. y Rodríguez, C. (2008). Las tutorías en el Espacio Europeo de Educación Superior: valoración de su implementación en la titulación de Maestro. Revista de Investigación Psicoeducativa, 6(1), 65-85.

- Hollenhorst, S., Olson, D. y Fortney, R. (1992). Use of importance-performance analysis to evaluate state park cabins: the case of the West Virginia state park system. Journal of Park and Recreation Administration, 10(1), 1-11.

- Jacobs, B. y Van Der Ploeg, F. (2006). Guide to reform of higher education: A European perspective. Economic Policy, 21(47), 535-592.

- Joseph, M. y Joseph, B. (1997). Service quality in education: a student perspective. Quality Assurance in Education, 5(1), 15-21.

- Lapidus, R. y Brown, J. (1993). Assesing Satisfaction with University Experience: An International Perspective. Journal of Consumer Satisfaction and Complaining Behaviour, 6, 187-195.

- Learreta, M. B., Arriaga, A. y Cruz, A. M. (2006). Nivel de satisfacción del alumnado de primero de la Facultad de Ciencias de la Actividad Física y del Deporte respecto a su aprendizaje. III Jornadas Internacionales de Innovación Universitaria, Villaviciosa de Odón (Madrid), 14-15 de septiembre.

- Letcher, D.W. y Neves J.S. (2010). Determinant of undergraduate business student satisfaction. Research in Higher Education Journal, 1-26.

- Martilla, J.A. y James, J. C. (1977). Importance-performance analysis. Journal of Marketing, 41(1), 77-79.

- Martínez J. A. (2018). La consideración de la propagación de errores en el análisis de importancia-valoración en gestión deportiva. Cuadernos De Psicología Del Deporte, 18(3), 141-149.https://revistas.um.es/cpd/article/view/325311

- Martínez-Caro, E., Martínez-Caro, E., y Díaz-SuardíazA. (2014). La calidad del servicio en los clubes de fútbol base de la región de Murcia. Una aplicación del análisis de importanciasatisfacción. SPORT TK-Revista EuroAmericana de Ciencias del Deporte, 3(1-2), 33-40.

- Mauri, T., Coll, C. y Onrubia, J. (2008). La evaluación de la calidad de los procesos de innovación docente universitaria. Una perspectiva constructivista. Red U. Revista de Docencia Universitaria, 1. http://red-u.net/redu/files/journals/1/articles/47/public/47-36-2PB.pdf

- Nale, R. D., Rauch, D. A., Wathen, S. A. y Barr, P.B. (2000). An exploratory look at the use of importance-performance analysis as a curricular assessment tool in a school of business. Journal of Workplace Learning, 12(4), 139-145.

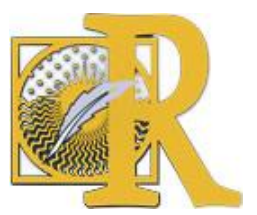


- Oliver, R. L. (1980). A cognitive model of the antecedents and consequences of satisfaction decisions. Journal of marketing Research, 17, 460-469.

- Oliver, R. L. y Desarbo, W. S. (1989). Processing satisfaction response in consumption: A suggested framework and response proposition. Journal of Consumer Satisfaction. Dissatisfaction and Complaining Behaviour, 1-16.

- Ortinau, D., Bush, A., Bush, R. y Twible, J. L. (1989). The use of Importance-Performance for improving the quality of marketing education: Interpreting Faculty-Course evaluations. Journal of Marketing Education, 11(2), 78-86.

- Pérez, J. A., Lozano, J.A., Gómez De Terreros, M. y Aguilera, A. (2010). Diseño de un instrumento para la evaluación de la satisfacción de la formación recibida de las diferentes asignaturas correspondientes al plan de estudios del Grado en Psicología de la Universidad de Sevilla. Revista de Enseñanza Universitaria, 36, 45-61.

- Petruzzellis, L., D'uggento, A. M., y Romanazzi, S. (2006). Student satisfaction and quality of service in Italian universities. Managing Service Quality, 16(4), 349-364.

- Picón, E., Varela, J. y Braña, T. (2011). La representación de los datos mediante el Análisis de Importancia-Valoración. Metodología de Encuestas, 13, 121-142.

- Pike, S.D. y Larkin, I. K. (2010). Longitudinal evaluations of student satisfaction with a postgraduate unit using importance-performance analysis. Journal of Teaching in Travel and Tourism, 10(3), 215-231.

- Sampson, S. E. y Showalter, M. J. (1999). The performance-importance response function: Observations and implications. Service Industries Journal, 19(3), 1-26.

- Seymour, D. T. (1993). On Q: Causing quality in higher education. Phoenix, AZ; Oryx. MacMillan Publishing Company.

- Serrano, V.M., Rial, A. y Carvalho, M.J. (2014). Análisis de importancia valoración (IPA) como herramienta de diagnóstico en la gestión de clubes de golf. Revista Intercontinental de Gestão Desportiva, 4 (1): 86-98.

- Serrano-Gómez, V., Rial, A., García-García, O. y Gambau, V. (2013). QGolf-9. Escala para la evaluación de la calidad percibida en los clubes de golf. Revista de Psicología del Deporte, 22 (1), 111- 121.

- Slack, N. (1994). The Importance-Performance Matrix as a Determinant of Improvement Priority. International Journal of Operations y Production Management, 14(5), 59-75.

- Tomusk, V. (2006). Creating the European Area of Higher Education: Voices from the periphery. Dordrecht, Países Bajos: Springer.

- Zamorano, S. y García, J. (2018). El análisis importancia-valoración según género y permanencia: el caso de los centros de fitness. Materiales para la Historia del Deporte, 16, 24-35.

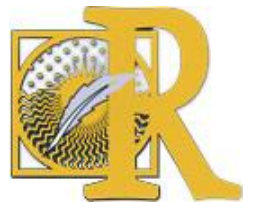

